\title{
Maximal and minimal solutions and comparison results for differential equations in abstract cones
}

\author{
by V. Lakshmikantham, A. Richard Mttchell and
}

Roger W. Mitchele (Arlington, Texas)

\begin{abstract}
Employing the properties of abstract cones and using the Kuratowski measure of non-compactness of a set, existence of extremal solutions of differential equations in a Banach space has been established. On the basis of these results, an abstract comparison theorem is proved and as an application of the comparison principle a general uniqueness theorem is considered.
\end{abstract}

1. Introduction. As is well known, an important technique in the theory of differential equations is concerned with estimating a function satisfying a differential inequality by means of the extremal solutions of the corresponding differential equation. This comparison principle has been widely employed in studying the qualitative theory of differential equations (see [3]).

If we desire to develop a similar comparison result in abstract spaces we must consider cones. These results could be of great value in applications to the theory of differential equations in abstract spaces. First, we must consider existence results for maximal and minimal solutions in cones which can then be utilized to prove comparison results. This approach would unify various comparison theorems for scalar, finite, and infinite systems of differential equations. Naturally the notion of quasi-monotone functions must be introduced for abstract spaces.

In this paper, employing the properties of abstract cones and the Kuratowski measure of non-compactness of a set, we prove existence of extremal solutions, discuss comparison theorems and, as an application of the comparison technique, consider a general uniqueness theorem.

2. Preliminary results and definitions. Let $B$ denote a real Banach space with norm $\|\cdot\|$. A cone, $K$, is a proper subset of $B$ such that if $v$, $w \in K, \lambda \in R$ with $\lambda>0$, then $v+w, \lambda v \in K$. Throughout this paper we 
will consider a closed cone $Q$ and its interior $P\left(P=Q^{0}\right)$. These cones induce orderings on $B$ defined by

and

$$
x \leqslant y \quad \text { if } \quad y-x \in Q,
$$

$$
x<y \quad \text { if } \quad y-x \in P .
$$

The following lemma states some fundamental properties of such cones (see [1]).

Lemma 1. Let $Q$ be a closed cone and $P$ an open cone such that $P=Q^{0}$. Then

$$
\begin{aligned}
& x \leqslant y, y<z \text { implies } x<z ; \\
& x \leqslant y, z<w \text { implies } x+z<y+w \\
& x_{n}<y_{n} \text { for each } n \text { and } x_{n} \rightarrow x, y_{n} \rightarrow y \text { implies } x \leqslant y \\
& x \in P \text { implies there exists } \lambda \in R \text { such that }
\end{aligned}
$$

$$
y<\lambda(\|y\|) x \text { for all } y \in B-\{0\} ;
$$

(1.5) $x \leqslant y$ and $x \nless y$ implies $y-x \in \partial(Q)=\partial(P)$, where for any set $A$, $\partial(A)$ denotes the boundary of $A$.

Let $P^{*}$ be the set of all continuous linear functionals, $c$, on $B$ such that $c(x)>0$ for all $x \in P$, and let $Q^{*}$ be the set of all continuous linear functionals, $c$, on $B$ such that $o(x) \geqslant 0$ for all $x \in Q$. A function $f$ from $B$ to $B$ is said to be quasi-monotone if $x \leqslant y$ and $c(x)=c(y)$ for some $e \in P^{*}$, then $c(f(x)) \leqslant c(f(y))$. This definition of quasi-monotone as well as the proof of the following basic result are given in [6].

THEOREM 2. Let $Q$ be a closed cone and $P$ an open cone such that $P=Q^{0}$. Assume that

(2.2) $f \in C\left[\left[t_{0}, t_{0}+a\right] \times B ; B\right]$ and $f$ is quasi-monotone with respect to $P$ for each $t$;

$$
\begin{aligned}
& D^{+} u(t)-f(t, u(t))<D^{+} v(t)-f(t, v(t)) \text { for all } t \in\left[t_{0}, t_{0}+a\right], \text { where } \\
& D^{+} u(t)=\varlimsup_{h \rightarrow 0^{+}} \frac{1}{h}[u(t+h)-u(t)] ;
\end{aligned}
$$

(2.4) $u\left(t_{0}\right)<v\left(t_{0}\right)$.

Then $u(t)<v(t)$ for all $t \in\left[t_{0}, t_{0}+a\right]$.

Let $a$ denote the Kuratowski measure of non-compactness (see [2]). We list below some fundamental properties of $\alpha$ which we will need (see [2], [4]). 
LEMMA 3. Let $S$ and $T$ be bounded subsets of a Banach space, B, and $\left\{a_{n}\right\}_{n=1}^{\infty}$ and $\left\{b_{n}\right\}_{n=1}^{\infty}$ be bounded sequences in $B$. Then

$$
\alpha(S+T) \leqslant \alpha(S)+\alpha(T), \text { where } S+T=\{s+t \mid s \in S \text { and } t \in T\}
$$

$a(\lambda S)=|\lambda| \alpha(S)$ for any scalar $\lambda$

$$
a\left(\left\{a_{n}\right\}_{n=1}^{\infty}\right)-a\left(\left\{b_{n}\right\}_{n=1}^{\infty}\right) \leqslant a\left(\left\{a_{n}-b_{n}\right\}_{n=1}^{\infty}\right)
$$

$\alpha(S)=0$ iff $\bar{S}$ is compact;

if $\|s\| \leqslant \varepsilon$ for all $s \in S$, then $a(S) \leqslant 2 \varepsilon$.

3. Existence of extremal solutions. We are now in a position to prove existence of maximal and minimal solutions for differential equations in abstract spaces. These results generalize in a natural way the corresponding results in finite dimensional spaces (see [3], [5]).

THEOREM 4. Let $Q$ be a closed cone and $P$ an open cone sueh that $P=Q^{0}$. Assume that

(4.1) $f \in C\left[\left[t_{0}, t_{0}+a\right] \times B, B\right]$, where $f$ is quasi-monotone with respec to $P$ for each $t$;

(4.2) $f$ is uniformly continuous on $\left[t_{0}, t_{0}+a\right] \times\left\{x \in B \mid\left\|x-x_{0}\right\| \leqslant b\right\}$, thust we may assume $a$ and $b$ are such that

$$
\|f(t, x)\| \leqslant M=\frac{b}{a} \quad \text { on } \quad\left[t_{0}, t_{0}+a\right] \times\left\{x \in B \mid\left\|x-x_{0}\right\| \leqslant b\right\}
$$

$$
\begin{aligned}
& g \in C\left[\left[t_{0}, t_{0}+a\right] \times R^{+}, R^{+}\right] \text {with } g(t, 0) \equiv 0, \text { and the system } u^{\prime} \\
& =g(t, u), u\left(t_{0}\right)=0 \text { has as its only solution } u \equiv 0 ;
\end{aligned}
$$

$a(\{x+h f(t, x) \mid x \in S\})-a(S) \leqslant h g(t, a(S))$ for $h>0, \quad S$ bounded, and $t \in\left[t_{0}, t_{0}+a\right]$.

Then there exists a maximal solution and a minimal solution of $x^{\prime}$ $=f(t, x), x\left(t_{0}\right)=x_{0}$ on the interval $\left[t_{0}, t_{0}+a / 2\right]$.

Proof. We will prove the existence of a maximal solution noting a similar proof yields existence of a minimal solution. Let $y_{0} \in P$ such that $\left\|y_{0}\right\|=\min \{b / 2, b / a\}$. For each positive integer $n$ consider the system

$$
x^{\prime}=f(t, x)+\frac{1}{n} y_{0}, \quad x\left(t_{0}\right)=x_{0}+\frac{1}{n} y_{0} .
$$

Applying Theorem 2 in [4] we have existence of a solution $x_{n}(t)$ of (4.5) for each $n$ and also a solution $x(t)$ of

$$
x^{\prime}=f(t, x), \quad x\left(t_{0}\right)=x_{0},
$$

where all solutions exist on $\left[t_{0}, t_{0}+a / 2\right]$.

Moreover, $\left\|x_{n}(t)-x_{0}\right\| \leqslant b$ and $\left\|x(t)-x_{0}\right\| \leqslant b$ for all $t \in\left[t_{0}, t_{0}+a / 2\right]$. 
By (4.2) and the choice of $y_{0}$ we have

$$
\left\|f(t, x)+\frac{1}{n} y_{0}\right\| \leqslant\|f(t, x)\|+\left\|\frac{1}{n} y_{0}\right\| \leqslant \frac{b}{a}+\frac{1}{n} \frac{b}{a} \leqslant 2 \frac{b}{a}=2 M
$$

on $\left[t_{0}, t_{0}+a\right] \times S_{b}\left(x_{0}\right)$. For $\varepsilon>0$ let $\delta=\min \{\varepsilon, \varepsilon / 2 M\}$.

If $t_{1}, t_{2} \in\left[t_{0}, t_{0}+a / 2\right]$ such that $\left|t_{1}-t_{2}\right|<\delta$, then

$$
\begin{aligned}
\left\|x_{n}\left(t_{1}\right)-x_{n}\left(t_{2}\right)\right\| & =\| \int_{t_{1}}^{t_{2}}\left(f\left(s, x_{n}(s)\right)+\frac{1}{n} y_{0}\right) d s \mid \\
& \leqslant\left|\int_{t_{1}}^{t_{2}}\right|\left|f\left(s, x_{n}(s)\right)+\frac{1}{n} y_{0}\right||d s| \\
& \leqslant\left|\int_{t_{1}}^{t_{2}} 2 M d s\right|=2 M\left|t_{1}-t_{2}\right|<\varepsilon .
\end{aligned}
$$

Thus, $\left\{x_{n}(t)\right\}_{n=1}^{\infty}$ is equicontinuous on $\left[t_{0}, t_{0}+a / 2\right]$.

For each $t \in\left[t_{0}, t_{0}+a / 2\right]$ define $m(t)=a\left(\left\{x_{n}(t)\right\}_{n=1}^{\infty}\right)$. The continuity of $m(t)$ on $\left[t_{0}, t_{0}+a / 2\right]$ follows easily from the properties of $a$ and the equicontinuity of $\left\{x_{n}(t)\right\}_{n=1}^{\infty}$. Now

$$
m\left(t_{0}\right)=\alpha\left(\left\{x_{n}\left(t_{0}\right)\right\}_{n=1}^{\infty}\right)=\alpha\left(\left\{x_{0}+\frac{1}{n} y_{0}\right\}_{n=1}^{\infty}\right)=0
$$

since $\left\{x_{0}+\frac{1}{n} y_{0}\right\}_{n=1}^{\infty}$ converges to $x_{0}$.

For $h>0$ and each $n$ we can write

$$
x_{n}(t+h)-x_{n}(t)=h f\left(t, x_{n}(t)\right)+h \varepsilon_{n}(h),
$$

where

$$
\lim _{h \rightarrow 0^{+}} a\left(\left\{\varepsilon_{n}(h)\right\}_{n=1}^{\infty}\right)=0 .
$$

To see this, let $\varepsilon>0$. By the uniform continuity of $f$ there exists $\delta>0$ such that $\left(t_{1}, y_{1}\right),\left(t_{2}, y_{2}\right) \in\left[t_{0}, t_{0}+a / 2\right] \times S_{b}\left(x_{0}\right)$ and $\left|t_{1}-t_{2}\right|+\left\|y_{1}-y_{2}\right\|<\delta$ implies $\left\|f\left(t_{1}, y_{1}\right)-f\left(t_{2}, y_{2}\right)\right\|<\varepsilon$. By the equicontinuity of $\left\{x_{n}(t)\right\}_{n=1}^{\infty}$ ther $\theta$ exists $\gamma$, where $0<\gamma<\delta / 2$ and such that $t_{1}, t_{2} \in\left[t_{0}, t_{0}+a / 2\right]$ and $\left|t_{1}-t_{2}\right|$ $<\delta$ implies $\left\|x_{n}\left(t_{1}\right)-x_{n}\left(t_{z}\right)\right\|<\delta / 2$ for each $n$. Thus, if $h<\gamma$ and $t, t+h$ $\in\left[t_{0}, t_{0}+a / 2\right]$ we have

$$
\begin{aligned}
\left\|h \varepsilon_{n}(h)\right\| & =\left\|x_{n}(t+h)-x_{n}(t)-h f\left(t, x_{n}(t)\right)\right\| \\
& =\left\|\int_{t}^{t+h}\left[f\left(s, \grave{x_{n}}(s)\right)-f\left(t, x_{n}(t)\right)\right] d s\right\| \\
& <\int_{t}^{t+h} \varepsilon d s=\varepsilon h \quad \text { for each } n .
\end{aligned}
$$


Hence, $\left\|\varepsilon_{n}(h)\right\|<\varepsilon$ for each $n$ and by (3.5) we have $a\left(\left\{\varepsilon_{n}(h)\right\}_{n=1}^{\infty}\right)<2 \varepsilon$. Now $\varepsilon$ was arbitrary and so $\lim _{h \rightarrow 0^{+}} a\left(\left\{\varepsilon_{n}(h)\right\}_{n=1}^{\infty}\right)=0$.

Using (4.8), (4.4), and (4.9) we have

$$
\begin{aligned}
D^{+} m(t) & =\varlimsup_{h \rightarrow 0^{+}} \frac{m(t+h)-m(t)}{h}=\varlimsup_{h \rightarrow 0^{+}} \frac{\alpha\left(\left\{x_{n}(t+h)\right\}_{n=1}^{\infty}\right)-\alpha\left(\left\{x_{n}(t)\right\}_{n=1}^{\infty}\right)}{h} \\
& =\varlimsup_{h \rightarrow 0^{+}} \frac{a\left(\left\{x_{n}(t)+h f\left(t, x_{n}(t)\right)+h\left(\left\{\varepsilon_{n}(h)\right\}_{n=1}^{\infty}\right)\right)-a\left(\left\{x_{n}(t)\right\}_{n=1}^{\infty}\right)\right.}{h} \\
& \leqslant \varlimsup_{h \rightarrow 0^{+}} \frac{\left[a\left(\left\{x_{n}(t)+h f\left(t, x_{n}(t)\right)\right\}_{n=1}^{\infty}\right)-a\left(\left\{x_{n}(t)\right\}_{n=1}^{\infty}\right)\right]+a\left(\left\{h \varepsilon_{n}(h)\right\}_{n=1}^{\infty}\right)}{h} \\
& \leqslant \varlimsup_{h \rightarrow 0^{+}} \frac{h g\left(t, a\left(\left\{x_{n}(t)\right\}_{n=1}^{\infty}\right)\right)}{h}+\varlimsup_{h \rightarrow 0^{+}} \frac{h \alpha\left(\left\{\varepsilon_{n}(h)\right\}_{n=1}^{\infty}\right)}{h} \\
& =g(t, m(t))+0 .
\end{aligned}
$$

Hence,

$$
D^{+} m(t) \leqslant g(t, m(t)) \quad \text { for } t \in\left[t_{0}, t_{0}+a / 2\right) .
$$

Using the Comparison Theorem (page 15 in [3]) and (4.10) we have $m(t) \leqslant r(t)$, where $r(t)$ is the maximal solution of $u^{\prime}=g(t, u), u\left(t_{0}\right)=0$ but by (4.3). the only solution is the zero solution. Thus, $m(t) \equiv 0$ on $\left[t_{0}, t_{0}+a / 2\right]$ and so $\left\{x_{n}(t)\right\}_{n=1}^{\infty}$ is relatively compact for each $t \in\left[t_{0}, t_{0}+a / 2\right]$.

Applying the Ascoli Theorem we obtain a subsequence (for simplicity of notation we will use the sequence itself) which converges uniformly to a continuous function $r(t)$ on $\left[t_{0}, t_{0}+a / 2\right]$. For each $t \in\left[t_{0}, t_{0}+a / 2\right]$

$$
x_{n}(t)=x_{n}\left(t_{0}\right)+\int_{t_{0}}^{t}\left[f\left(s, x_{n}(s)\right)+\frac{1}{n} y_{0}\right] d s .
$$

Using uniform convergence and taking limits we obtain $r(t)=x_{0}+$ $+\int_{t_{0}}^{t} f(s, r(s)) d s$, so $r(t)$ is a solution of $(4.6)$ on $\left[t_{0}, t_{0}+a / 2\right]$. Let $x(t)$ be any solution of $(4.6)$. Now,

$$
x^{\prime}-f(t, x)=0<\frac{1}{n} y_{0}=x_{n}^{\prime}-f\left(t, x_{n}\right)
$$

and

$$
x\left(t_{0}\right)=x_{0}<x_{0}+\frac{1}{n} y_{0}=x_{n}\left(t_{0}\right)
$$

Applying Theorem 2 we have $x(t)<x_{n}(t)$ for all $t \in\left[t_{0}, t_{0}+a / 2\right]$ so $x(t)$ $\leqslant \lim _{n \rightarrow \infty} x_{n}(t)=r(t)$ for all $t \in\left[t_{0}, t_{0}+a / 2\right]$. Thus, $r(t)$ is the desired maximal solution. 
4. Comparison results. Having the existence results for maximal solutions at our disposal, it is easy to prove a general comparison result for abstract spaces. Obviously such a result should and does contain the well-known comparison theorems for scalar and vector cases (see [3]). Also, this result includes a comparison theorem for infinite systems (see [5]).

THEOREM 5. Consider the system $x^{\prime}=f(t, x), x\left(t_{0}\right)=x_{0}$ and suppose the hypotheses of Theorem 4 are satisfied. If $u \in C\left[\left[t_{0}, t_{0}+a / 2\right], B\right]$ such that $D_{-} u \leqslant f(t, u)$ on $\left[t_{0}, t_{0}+a / 2\right]$ and $u\left(t_{0}\right) \leqslant x_{0}$, then $u(t) \leqslant r(t)$ for all $t \in\left[t_{0}, t_{0}+a / 2\right]$, where $r(t)$ is the maximal solution of $x^{\prime}=f^{\prime}(t, x), x\left(t_{0}\right)=x_{0}$.

Proof. Let $x_{n}(t)$ be as in the proof of Theorem 4. Notice $u\left(t_{0}\right) \leqslant x_{0}$ $<x_{0}+\frac{1}{n} y_{0}=x_{n}\left(t_{0}\right)$ and $D_{-} u(t)-f(t, u(t)) \leqslant 0<\frac{1}{n} y_{0}=x_{n}^{\prime}(t)-f\left(t, x_{n}(t)\right)$ for all $t \in\left[t_{0}, t_{0}+a / 2\right]$. By Theorem 2, $u(t)<x_{n}(t)$ for each $n$ and for all $t \in\left[t_{0}, t_{0}+a / 2\right]$. Thus, $r(t)=\lim _{n \rightarrow \infty} x_{n}(t) \geqslant u(t)$ for all $t \in\left[t_{0}, t_{0}+a / 2\right]$.

Remark. Theorem 5 is a broad generalization of the comparison theorems. However, the more natural generalization which is useful in applications is where the range of $u$ is restricted to be in $Q$. In this situation one can prove a general result which includes the Bellman-Gronwall Lemma and its generalizations in finite dimensional spaces (see [3]).

Theorem 6. Let $Q$ be a closed cone and $P$ an open cone such that $P=Q^{0}$. Assume

(6.2) $g \in C\left[\left[t_{0}, t_{0}+a\right] \times B, B\right]$ such that $g(t, u)$ is non-decreasing (with respect to $Q$ ) in $u$ for each $t$;

there exists a maximal solution $r\left(t, t_{0}, u_{0}\right)$ of

$$
u^{\prime}=g(t, u), \quad u\left(t_{0}\right)=u_{0} \quad \text { on }\left[t_{0}, t_{0}+a\right]
$$

$$
v(t) \leqslant v\left(t_{0}\right)+\int_{i_{0}}^{t} g(s, v(s)) d s \text { for } t \in\left[t_{0}, t_{0}+a\right]
$$

$$
v\left(t_{0}\right) \leqslant u\left(t_{0}\right)=u_{0} .
$$

Then $v(t) \leqslant u(t)$ on $\left[t_{0}, t_{0}+a\right]$.

Proof. Let $w(t)=v\left(t_{0}\right)+\int_{t_{0}}^{t} g(s, v(s)) d s$ and note that $w\left(t_{0}\right)=v\left(t_{0}\right)$ $\leqslant u\left(t_{0}\right)=u_{0}$ and by (6.4) we have

$$
v(t) \leqslant w(t) \quad \text { for } t \in\left[t_{0}, t_{0}+a\right] .
$$

Now, $D_{-} w(t)=g(t, v(t))$ and so applying (6.2) we have $D_{-} w(t)=g(t, v(t))$ $\leqslant g(t, w(t))$ on $\left[t_{0}, t_{0}+a\right]$. By Theorem $5 w(t) \leqslant r(t)$ on $\left[t_{0}, t_{0}+a\right]$ and combining this and (6.6) we obtain $v(t) \leqslant r(t)$ on $\left[t_{0}, t_{0}+a\right]$. 
5. Application to uniqueness. In order to give an application of the comparison result to obtain a general uniqueness theorem we need to introduce the concept of a cone metric. Again, $Q$ will be a closed cone and $P$ an open cone with $P=Q^{0}$, but we will require $0 \in Q$ and if $u \in Q-\{0\}$, then $-u \notin Q$.

A function $\varrho: B \times B \rightarrow Q$ satisfying

and

$$
\begin{array}{ll}
\varrho(x, y)=0 & \text { iff } x=y, \\
\varrho(x, y)=\varrho(y, x) & \text { for all } x, y \in B,
\end{array}
$$

$$
\varrho(x, y) \leqslant \varrho(x, z)+\varrho(z, y) \quad \text { for all } x, y, z \in B \text {, }
$$

is called a $Q$-metric. In addition, we will assume that

and

$$
\varrho \in C[B \times B, Q],
$$

$$
\varrho(\lambda x, \lambda y)=|\lambda| \varrho(x, y), \quad \text { where } \lambda \in R
$$

$$
\varrho(x+z, y+z)=\varrho(x, y) .
$$

These additional restrictions seem reasonable when usual metrics on $R^{n}$ are considered.

Theorem 7 . Consider the system

$$
x^{\prime}=f(t, x), \quad x\left(t_{0}\right)=x_{0},
$$

where $f \in C\left[\left[t_{0}, t_{0}+a\right] \times\left\{x \in B \mid\left\|x-x_{0}\right\|<b\right\}\right]$. Assume that $g \in C\left[\left[t_{0}, t_{0}+a\right] \times\right.$ $\times B, B], g$ satisfies the hypotheses of Theorem 4 , and $u^{\prime}=g(t, u), u\left(t_{0}\right)=0$ has a unique solution, $u \equiv 0$. If $\varrho(f(t, x), f(t, y)) \leqslant g(t, \varrho(x, y))$ for all $(t, x)$, $(t, y) \in\left[t_{0}, t_{0}+a\right] \times\left\{x \in B \mid\left\|x-x_{0}\right\| \leqslant b\right\}$, then there is at most one solution for (7.1) on $\left[t_{0}, t_{0}+a / 2\right]$.

Proof. Let $x(t)$ and $y(t)$ be solutions of $x^{\prime}=f(t, x), x\left(t_{0}\right)=x_{0}$ and define $m(t)=\varrho(x(t), y(t))$ and note $m(t) \in Q$. Using the properties of $\varrho$. it is clear that $m(t)$ is continuous and we obtain

$$
\begin{aligned}
m(t+h)-m(t) & =\varrho(x(t)+h f(t, x(t))+h \varepsilon, y(t)+h f(t, y(t))+h \hat{\varepsilon})- \\
& =\varrho(x(t)-y(t), h f(t, y(t))-h f(t, x(t))+h(\hat{\varepsilon}-\varepsilon))- \\
& \leqslant \varrho(x(t)-y(t), 0)+\varrho(0, h f(t, y(t))-h f(t, x(t))+h(\hat{\varepsilon}-\varepsilon))- \\
& =\varrho(h(\varepsilon-\hat{\varepsilon}), h f(t, y(t))-h f(t, x(t))) \\
& \leqslant \varrho(h(\varepsilon-\hat{\varepsilon}), 0)+\varrho(0, h f(t, y(t))-h f(t, x(t))) \\
& =h \varrho(\varepsilon-\hat{\varepsilon}, 0)+h \varrho(f(t, x(t)), f(t, y(t))) .
\end{aligned}
$$


Thus

$$
\begin{aligned}
D^{+} m(t) & \leqslant \varlimsup_{h \rightarrow 0^{+}} \frac{h \varrho(\varepsilon-\hat{\varepsilon}, 0)+h \varrho(f(t, x(t)), f(t, y(t)))}{h} \\
& =\varlimsup_{h \rightarrow 0^{+}} \varrho(\varepsilon-\hat{\varepsilon}, 0)+\varlimsup_{h \rightarrow 0^{+}} \varrho(f(t, x(t)), f(t, y(t))) \\
& =\varrho(f(t, x(t)), f(t, y(t))) \leqslant g\left(t, \varrho^{\prime}(x(t), y(t))\right) \\
& =g(t, m(t)) .
\end{aligned}
$$

Moreover, $m\left(t_{0}\right)=\varrho\left(x\left(t_{0}\right), y\left(t_{0}\right)\right)=0$ so by Theorem 5 we know $m(t)$ $\leqslant r\left(t, t_{0}, 0\right)$ on $\left[t_{0}, t_{0}+a / 2\right]$, where $r\left(t, t_{0}, 0\right)$ is the maximal solution of $u^{\prime}=g(t, u), u\left(t_{0}\right)=0$. However, the only solution of $u^{\prime}=g(t, u), u\left(t_{0}\right)=0$ is $u \equiv 0$ so $m(t) \leqslant 0$ on $\left[t_{0}, t_{0}+a / 2\right]$ which means $-m(t) \in Q$. But $m(t) \in Q$ and $-m(t) \in Q$ is possible only if $m(t)=0$. Thus $m(t)=0$ on $\left[t_{0}, t_{0}+a / 2\right]$ which implies $x(t)=y(t)$ on $\left[t_{0}, t_{0}+a / 2\right]$ :

\section{References:}

[1] M. G. Krein, On positive definite funotionals in linear normed spaces, Amer. Math. Soc. Transl. of Math. Monographs 2, Providence (1962), p. 154-174.

[2] K. Kuratowaki, Topology, Vol. II, Academic Press, New York 1966.

[3] V. Lakshmikantham and S. Leela, Differential and integral inequalities, Vol. I, Academic Press, New York 1969.

[4] Tien Yien Li, Existence of solutions for ordinary differential equations in Banach spaces, to appear.

[5] W. Mlak and C. Olech, Integration of infinite systems of differential equations, Ann: Polon. Math. 13 (1963), p. 105-112.

[6] P. Volkmann, Gowöhnliche Differentialungleichungen mit Quasimonoton Wachsenden Funktionen in Topologischen Vektorraumen, Math. Zeitschr. 127 (1972), p. 157-164.

THE UNIVERSITY OF TEXAS AT ARLINGTON ARIINGTON, TEXAS

Reçu par la Rédaction le 7. 5. 1975
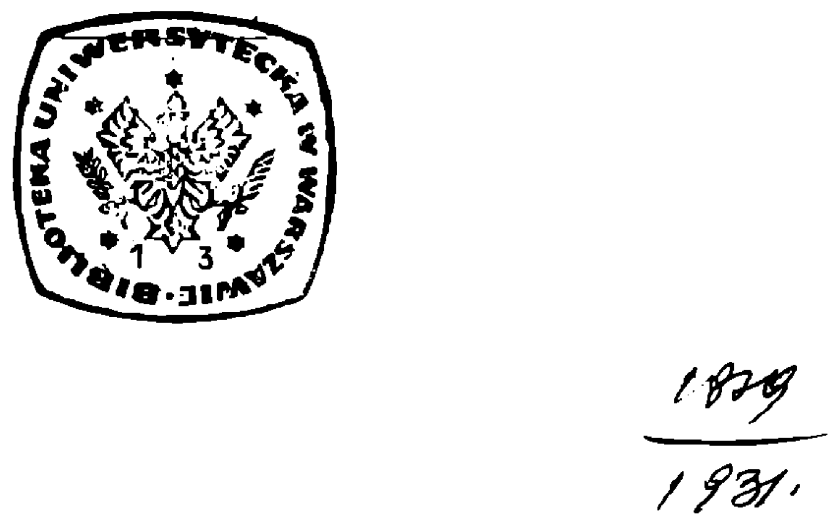